\title{
In vivo label-free mapping of the effect of a photosystem II inhibiting herbicide in plants using chlorophyll fluorescence lifetime
}

\author{
Elizabeth Noble ${ }^{1,2,3^{*}} \mathbb{D}$, Sunil Kumar ${ }^{1}$, Frederik G. Görlitz ${ }^{1}$, Chris Stain ${ }^{4}$, Chris Dunsby ${ }^{1,5 \neq}$ and Paul M. W. French ${ }^{1 \neq}$
}

\begin{abstract}
Background: In order to better understand and improve the mode of action of agrochemicals, it is useful to be able to visualize their uptake and distribution in vivo, non-invasively and, ideally, in the field. Here we explore the potential of plant autofluorescence (specifically chlorophyll fluorescence) to provide a readout of herbicide action across the scales utilising multiphoton-excited fluorescence lifetime imaging, wide-field single-photon excited fluorescence lifetime imaging and single point fluorescence lifetime measurements via a fibre-optic probe.

Results: Our studies indicate that changes in chlorophyll fluorescence lifetime can be utilised as an indirect readout of a photosystem II inhibiting herbicide activity in living plant leaves at three different scales: cellular $(\sim \mu \mathrm{m})$, single point $\left(\sim 1 \mathrm{~mm}^{2}\right)$ and macroscopic $\left(\sim 8 \times 6 \mathrm{~mm}^{2}\right.$ of a leaf). Multiphoton excited fluorescence lifetime imaging of Triticum aestivum leaves indicated that there is an increase in the spatially averaged chlorophyll fluorescence lifetime of leaves treated with Flagon EC - a photosystem II inhibiting herbicide. The untreated leaf exhibited an average lifetime of $560 \pm 30$ ps while the leaf imaged $2 \mathrm{~h}$ post treatment exhibited an increased lifetime of $2000 \pm 440 \mathrm{ps}$ in different fields of view. The results from in vivo wide-field single-photon excited fluorescence lifetime imaging excited at $440 \mathrm{~nm}$ indicated an increase in chlorophyll fluorescence lifetime from 521 ps in an untreated leaf to 1000 ps, just 3 min after treating the same leaf with Flagon EC, and to $2150 \mathrm{ps}$ after $27 \mathrm{~min}$. In vivo single point fluorescence lifetime measurements demonstrated a similar increase in chlorophyll fluorescence lifetime. Untreated leaf presented a fluorescence lifetime of $435 \mathrm{ps}$ in the $440 \mathrm{~nm}$ excited chlorophyll channel, $\mathrm{CH} 4$ (620-710 nm). In the first 5 min after treatment, mean fluorescence lifetime is observed to have increased to $1 \mathrm{~ns}$ and then to $1.3 \mathrm{~ns}$ after $60 \mathrm{~min}$. For all these in vivo plant autofluorescence lifetime measurements, the plants were not dark-adapted.

Conclusions: We demonstrate that the local impact of a photosystem II herbicide on living plant leaves can be conveniently mapped in space and time via changes in autofluorescence lifetime, which we attribute to changes in chlorophyll fluorescence. Using portable fibre-optic probe instrumentation originally designed for label-free biomedical applications, this capability could be deployed outside the laboratory for monitoring the distribution of herbicides in growing plants.
\end{abstract}

Keywords: Fluorescence spectroscopy, Plant, FLIM, Herbicide, Photosystem II, Chlorophyll fluorescence lifetime

\section{Background}

Understanding the specific fates of agrochemicals in plants is crucial to optimise their function. The

\footnotetext{
*Correspondence: e.noble@imperial.ac.uk

${ }^{\dagger}$ Chris Dunsby and Paul M.W. French contributed equally to this work

${ }^{2}$ Department of Chemistry, Imperial College London,

London SW7 2AZ, UK

Full list of author information is available at the end of the article
}

absorption, distribution, transportation and storage of agrochemicals within and through the various cellular structures will greatly affect the extent and effectiveness of their interaction with plant metabolism [1] and so the ability to map the distribution or effect of agrochemicals in vivo could help optimise the formulation of new agrochemicals.

In vitro methods that are based on solvent extraction or cell culture analysis, such as biochemical assays or mass 
spectrometry $[2,3]$, can provide highly specific chemical information but are destructive and time consuming. Furthermore, any physical or chemical perturbation to the natural state of a plant can result in stress, potentially triggering various reflex mechanisms that could lead to incorrect inferences [4] and such measurements can fail to report the true spatio-temporal distribution and interaction of agrochemicals. Analytical methods such as radiolabelling [5] can provide highly specific information on herbicide metabolism and over all absorption rates, but do not offer spatially resolved herbicide distribution data. Scanning electron microscopy (SEM) [6] can provide higher spatial resolution but can be prone to artefacts arising from sample fixation and mechanical sectioning and cannot follow dynamic events.

Fluorescence imaging techniques, such as widefield fluorescence imaging [7], confocal laser scanning microscopy (CLSM) $[8,9]$ and two-photon excitation microscopy (TPEM) $[10,11]$, provide the ability to follow dynamic events, including in live plants. Hence, it is desirable to explore non-invasive fluorescence imaging techniques, capable of providing qualitative and quantitative information concerning the in vivo distribution of agrochemicals, in the laboratory and in the field. In principle, this could be realised by fluorescence imaging of agrochemicals that are intrinsically fluorescent. However, it is generally challenging to distinguish such fluorescence from background autofluorescence arising from chlorophyll and/or other endogenous fluorophores. Direct fluorescent labelling of the agrochemical could improve the contrast but such labels are comparable to or larger in size than the agrochemicals themselves and may compromise their mode of action. An alternative approach is to map the local action of an agrochemical through its impact on the intrinsic (chlorophyll) fluorescence and thereby infer information concerning the agrochemical distribution. Here we demonstrate this approach using fluorescence lifetime imaging of chlorophyll to map the local action of a PS II inhibiting herbicide.

Herbicides represent a major fraction of all agrochemicals used, of which PS II inhibiting herbicides are an important class. Light energy absorbed by leaves can be expended driving the photochemistry of photosynthesis, emitted as chlorophyll fluorescence or dissipated as heat [12]. When a chlorophyll molecule absorbs light and is promoted to its first singlet excited state, the excited state energy can be transferred to other chlorophyll molecules and ultimately to the photosynthetic reaction centres (PS II and PS I) through Förster resonance energy transfer [13]. The presence of a photosynthetic inhibitor suppressing this pathway leads to an increase in the lifetime of singlet excited chlorophyll, which increases the probability of inter-system crossing to long lived chlorophyll triplet excited states. This increases the probability of transfer of energy from the triplet excited excited states of chlorophyll to ground state molecular oxygen, which results in the formation of singlet state molecular oxygen that can subsequently lead to the production of radical species such as ${ }^{3} \mathrm{Chl},{ }^{1} \mathrm{O}_{2}, \mathrm{H}_{2} \mathrm{O}_{2}$ and $\mathrm{O}_{2}{ }^{-}$. These radicals are phytotoxic in nature and can hinder important biological processes or cause membrane damage. Treating a plant with a PS II inhibitor will trigger an increased production of such radical species and can result in phytotoxicity. This is the basis for the mode of action of most of PS II inhibiting herbicides [14].

Chlorophyll fluorescence can thus provide a readout of the action of PS II inhibitors. In general, the balance between photochemistry, chlorophyll fluorescence and heat dissipation following the absorption of light has been studied using a range of chlorophyll fluorometric techniques that are sensitive to the photosynthetic electron transfer rate and therefore provide information on the overall PS II efficiency [15]. Early studies were based on the assumption that the light energy absorbed in leaves was divided equally between the PS I and PS II light harvesting complexes [15] and therefore the factor, $F_{v} / F_{m}$, which is the ratio of fluorescence variation to maximal fluorescence, gives a measure of maximum PS II efficiency [16]. Note that $F_{v}=F_{m}-F_{o}$, where $F_{o}$ is the fluorescence from the leaf in the presence of weak measuring light $\left(\sim 0.1 \mu \mathrm{mol}\right.$ photons $\left.\mathrm{m}^{-2} \mathrm{~s}^{-1}\right)$ and $F_{m}$ is the maximum fluorescence from a dark-adapted leaf when excited by a saturating light flash, i.e. one with sufficient intensity to drive a high proportion of the PSII centres into the "closed" state where they are not capable of photochemistry. For Arabidopsis, a saturating photon flux density of $\sim 4000 \mu \mathrm{mol}$ photons $\mathrm{m}^{-2} \mathrm{~s}^{-1}$ is required [17] but this value may vary between plant species. Subsequent studies, however, showed that this assumption was not valid due to PS I fluorescence varying independently of photosynthetically active photon flux density, unlike PS II [15]. Furthermore, the PSI and PSII fluorescence vary differently as a function of temperature [18]. Therefore estimates of the photosynthetic electron transfer rate based on measurements of photosynthetic yield, $F_{v} / F_{m}$ [17] could be erroneous. Nevertheless, chlorophyll fluorescence-based approaches have been successfully applied at different scales to study the dynamics of basic photosynthetic reactions, including biotic/abiotic stress responses [19-21], and efforts are being made to translate this optical signal from laboratory to field phenotyping [22] in plants, linking microscopic observations to macroscopic and to leaf level dynamics of photosynthetic reactions. Chlorophyll fluorescence imaging has previously been used to obtain qualitative readouts of metabolic changes correlated to herbicide action, as 
reported in [17], although the instrumentation used could not localise herbicide distribution with high resolution. Higher resolution fluorescence imaging studies $[15,23,24]$, have demonstrated that the uptake of the herbicide Diuron can be monitored in plant leaves using chlorophyll fluorescence imaging following dark adaption, although these utilised wide-field imaging studies and so did not provide depth-resolved imaging of changes to chlorophyll fluorescence. Optically sectioned imaging can be provided by confocal laser scanning fluorescence microscopy and used to image up to a depth of $100-150 \mu \mathrm{m}$ but image quality deteriorates when imaging deeper than $\sim 100 \mu \mathrm{m}$ into the sample owing to background fluorescence and scattering effects caused by the leaf tissues [25]. Improved performance in terms of imaging depth and reduced background fluorescence can be provided by two photon excitation, due to the increased ability of near-infrared light to penetrate biological samples and the limiting of excitation to the focal plane, as has been applied in plant leaves e.g. [26]. However, optical scattering and aberrations still impact the quantification of intensity-based chlorophyll fluorescence readouts.

Fluorescence lifetime measurements [27] can provide quantitative readouts even when image information is degraded and absolute intensity measurements are compromised by optical scattering, sample absorption (inner filter effect) and/or variations in fluorophore concentration. Fluorescence lifetime provides a direct readout of the impact of the local fluorophore environment on relaxation pathways following excitation and so provides powerful sensing capabilities. In biomedicine, fluorescence lifetime measurements and fluorescence lifetime imaging (FLIM) are used to study changes in tissue autofluorescence [28]. However, fluorescence lifetime techniques have not been widely utilized for plant studies although FLIM has been used to study photosynthesis $[26,29,30]$, and the uptake of minerals [31, 32] and was previously used to study the effect of a photosynthetic inhibitor DCMU (3-(3,4-dichlorophenyl)-1,1-dimethylurea) in Chlamydomonas reinhardtii [29]. We report here the application of fluorescence lifetime imaging of chlorophyll to provide a label-free in vivo means to non-invasively map the effect of a PS II inhibiting herbicide in Triticum aestivum (Winter Wheat) on different spatial scales through its local impact on chlorophyll fluorescence.

The action of inhibitors on the photosynthetic electron transport chain has previously been observed using non-imaging, time-resolved fluorescence spectroscopy techniques: Petrasek et al. [33] observed an overall increase in chlorophyll fluorescence lifetime under the stress of a PS II inhibitor, DCMU, and Hunsche et al. [34] reported a significant increase in the mean lifetime of the fluorescence measured from plants treated with PS II inhibiting herbicides. FLIM experiments have also shown that the inhibition of PS II by a herbicide leads to an increase of the chlorophyll fluorescence intensity and lifetime-attributed to a reduction in photochemical quenching-but no spatially resolved lifetime data was presented [26].

Here we explore the potential to utilise the change in chlorophyll fluorescence lifetime as an indirect read out of herbicide activity and thereby map the time-dependent uptake of the herbicide by plants in vivo-and ultimately in the field-using FLIM or single-point probe lifetime measurements. We present in vivo readouts from non-dark adapted plants at three different scales: cellular $(\sim \mu \mathrm{m})$, single point $\left(\sim 1 \mathrm{~mm}^{2}\right)$ and macroscopic $\left(\sim 8 \times 6 \mathrm{~mm}^{2}\right.$ of a leaf $)$ to study the distribution of the effect of a PS II inhibiting herbicide. We first utilised twophoton laser scanning microscopy (TPLSM) to perform in vivo FLIM in leaves excited at $900 \mathrm{~nm}$, taking advantage of the deeper optical sectioning and reduced out-ofplane photobleaching and phototoxic effects compared to single photon laser scanning confocal microscopy [35]. While it is difficult to make a generalization of the typical imaging depth achievable, since plant leaves have a complex structural anatomy that varies from species to species, we note that TPLSM imaging depths of $\sim 200 \mu \mathrm{m}$ have been reported $[10,36]$. We also explored the potential to repurpose instrumentation originally developed for autofluorescence lifetime studies of human tissue for medical applications, noting that this instrumentation is already relative portable and could be engineered for application in the field. Specifically, we applied a custombuilt time-resolved spectrofluorometer, which was originally developed to make single point measurements of tissue via a fibre-optic probe [37] to make measurements of plant leaves treated and untreated with the PSII inhibiting herbicide, Flagon 400 EC. This approach can provide mapping of the action of herbicides with a spatial resolution on the order of millimetres. Finally, for macroscopic imaging, we applied wide-field time-gated FLIM technology [38], obtaining maps of herbicide action on a time scale of seconds. We note that spectrally resolved lifetime measurements can provide further contrast, which could address the interference from other plant pigments, e.g. [39, 40].

\section{Methods}

\section{Plant material and growth conditions}

For the studies conducted, plants of Triticum aestivum (Winter Wheat cv. Hereward), a cool climate monocot food crop (Growth room conditions: $20 / 16{ }^{\circ} \mathrm{C}$ (day/night temp) $16 \mathrm{~h}$ of daylight, approx. $65 \%$ relative humidity, lighting- $150 \mu \mathrm{mol} / \mathrm{m}^{2} / \mathrm{s}$ or $31.2 \mathrm{~W} / \mathrm{m}^{2}$ ) were used. Seeds 
were obtained from Syngenta Jealott's Hill International Research Centre Bracknell, Berkshire, UK.

\section{Chemicals}

PS II inhibiting herbicide Flagon 400 EC was provided in solution by Syngenta (Jealott's Hill International Research Centre Bracknell, Berkshire, UK) and was diluted in milliQ water.

\section{Sample preparation and plant treatment}

Before the plants are treated with active ingredient (AI) formulation, a non-fluorescent felt pen (Berol Toughpoint) was used to mark the desired treatment area with four spots on a square with side approximately $10 \mathrm{~mm}$ so that the treated area could be easily located again in subsequent fluorescence measurements. Flagon EC 400 was diluted in water to a concentration of $\sim 49 \mathrm{ppm} / 1.22 \mathrm{M}$ and two closely spaced $2 \mu \mathrm{l}$ droplets (a close approximation to the the size of typical spray droplets used in the field) were applied using a Hamilton micropipette (Hamilton Bonaduz AG, Bonaduz, Switzerland) in the centre of the four marked spots.

For measuring the leaf autofluorescence excitation/ emission matrix, a new leaf was used for each emission spectrum to avoid any photochemical changes to the leaf caused by the excitation light. For each set of experiments, all the leaves were from plants of the same age and grown under the same growth conditions. Unless otherwise specified, we used 18 days old plants for the measurements reported here.

\section{Measurement of fluorescence spectra}

The autofluorescence of Triticum aestivum plant leaves and also the fluorescence properties of the herbicide were characterised in order to optimise the excitation wavelengths and fluorescence detection spectral windows for these studies. The fluorescence excitation and emission spectra of Flagon EC 400 and the plant leaves were measured using a commercial UV/VIS Spectrofluorometer (RF-5301PC, Shimadzu, Japan).

\section{Characterisation of Flagon 400 EC fluorescence}

Time-resolved fluorescence measurements of Flagon EC 400 were undertaken using the custom-built multidimensional spectrofluorometer described in [41], of which a schematic diagram of the optical set-up of the system is provided in Additional file 1. A supercontinuum laser source (SPC-400, $20 \mathrm{MHz}$ Fianium, UK) operating at a repetition rate of $20 \mathrm{MHz}$ provides tunable picosecond pulses for excitation. For measurements of Flagon EC 400, a bandpass filter centered at 400 and $40 \mathrm{~nm}$ bandwidth was used to select the excitation radiation that passes through a polarizer before reaching sample solution in a cuvette. The detection beam path is at right angles to the excitation beam path and the resultant fluorescence decay is recorded for 11 distinct emission wavelengths spaced $2 \mathrm{~nm}$ apart over the range $480-500 \mathrm{~nm}$, using time-correlated single photon counting (TCSPC), with an average of $\sim 6000$ photon counts over an acquisition time of $180 \mathrm{~s}$ per decay. All measurements were performed with a polarizer in the emission path placed at the magic angle polarization to remove any fluorescence anisotropy effects. The whole system is controlled by a custom-written LabVIEW software (LabVIEW, National Instruments). The instrument response function (IRF) for the fluorometer is measured using a scatterer, LUDOX (a solution of colloidal silica, Sigma-Aldrich, UK). The fluorescence decay curves are analysed using the FLIMfit software tool developed at Imperial College London [42] and the maximum likelihood iterative method was used to fit the experimental data to model fluorescence decay profiles.

\section{Multiphoton excited fluorescent lifetime imaging (MPE-FLIM)}

MPE-FLIM in plant cells was undertaken both in vivo in a live plant leaf and in situ in a recently removed plant leaf in order to obtain high (subcellular) resolution maps of chlorophyll fluorescence lifetime. FLIM measurements were made using a Leica SP5 system (TCS SP5, Leica Microsystems GmbH, Germany) with a tunable (690$1020 \mathrm{~nm}$ ) Ti:Sapphire laser (Spectra-Physics, Broadband Mai Tai) providing $100 \mathrm{fs}$ pulses at $80 \mathrm{MHz}$ for multiphoton excitation and FLIM implemented with TCSPC. The optimum excitation wavelength for mulitphoton imaging of chlorophyll in a plant leaf was determined using a detection band of $680-735 \mathrm{~nm}$ and by scanning the excitation wavelength over the range $850-990 \mathrm{~nm}$. The highest signal was obtained for excitation at $900 \mathrm{~nm}$ and so this was chosen for all subsequent multiphoton imaging. Treated and untreated leaf samples were imaged in situ and in vivo using multiphoton excitation at $900 \mathrm{~nm}$ and detection in the band $600-730 \mathrm{~nm}$, with typical acquisition times of $40 \mathrm{~s}$ being required to acquire images with $256 \times 256$ pixels. The leaves were treated with two closely spaced $2 \mu \mathrm{l}$ droplets of Flagon EC 400 and were imaged after $2.5 \mathrm{~h}$ for the in situ experiment. For in vivo time course experiments, images were taken at $5 \mathrm{~min}$ intervals starting from $5 \mathrm{~min}$ after treatment to $45 \mathrm{~min}$.

The instrument response function (IRF) of the system was recorded using gold nano-rods and a background image (with no excitation) with the same experimental parameters as FLIM measurements. The fluorescence decay data were analyzed using the FLIMfit software [42]. 


\section{Multispectral point-probe fluorescence lifetime measurements in vivo}

To explore the potential to monitor herbicide distribution in the field, we used a portable time-resolved spectrofluorometer incorporating a fibre-optic probe that was originally developed for clinical and preclinical studies [37], to make single point measurements of leaves on live plants. This instrument, which is depicted in Fig. 1, incorporates two picosecond pulsed excitation diode lasers: a laser diode (LDH-P-C-375B, PicoQuant GmbH, Germany) that provides $70 \mathrm{ps}$ pulses at $372 \mathrm{~nm}$ with an average output power of $3.3 \mathrm{~mW}$ and a laser diode (LDHP-C-440B, PicoQuant GmbH, Germany) providing 90 ps pulses at $440 \mathrm{~nm}$ with $3.5 \mathrm{~mW}$ average power. The repetition rates of both the lasers were set to $20 \mathrm{MHz}$. The laser beams are coupled into a custom-made optical fiber bundle (FiberTech Optica, Canada) consisting of three excitation fibers and fourteen detection fibers arranged in a hexagonal structure around the excitation fibers.

For these experiments the fibre-optic probe was held by a clamp to the leaf and fluorescence was collected by the detection fibers into three spectrally resolved detection channels implemented using a set of dichroic mirrors and band-pass filters. The leaves were treated with two drops $(2 \mu \mathrm{l})$ of the herbicide Flagon EC 400 and measurements were made 5 min afterwards. At $372 \mathrm{~nm}$ excitation, all the three channels are used: the first channel collects light from 400 to $420 \mathrm{~nm}$ (CH1); the second channel collects fluorescence light from 430 to $480 \mathrm{~nm}$ (CH2); and the third channel collects light from 620 to $710 \mathrm{~nm}(\mathrm{CH} 3)$. For the $440 \mathrm{~nm}$ excitation light, only the third (620-710 $\mathrm{nm})$ channel is active and this is referred as "channel 4" (CH4). Since $440 \mathrm{~nm}$ is an optimum excitation wavelength for chlorophyll fluorescence in leaves, $\mathrm{CH} 4$ is the spectral channel of primary interest for this study. The integration time for each acquisition was about $30 \mathrm{~s}$ for both UV excitation as well as 440 $\mathrm{nm}$ excitation. $1.7 \mu \mathrm{W}$ of $375 \mathrm{~nm}$ excitation and $29 \mu \mathrm{W}$ of $440 \mathrm{~nm}$ excitation at the distal tip of the fibre probe were used for measurements of plant leaves. IRF measurements were made for each detection channels using a scattering sample under the same conditions. This fibreoptic probe interrogates an area of $\sim 1 \mathrm{~mm}^{2}$ on the leaf and so can provide $\mathrm{mm}$ spatial resolution.

\section{In vivo wide-field macroscopic time-gated fluorescence imaging}

For imaging herbicide distribution with higher spatial resolution and on faster timescales, we constructed a wide field FLIM macroscope for in vivo imaging of plants. A schematic diagram describing the setup is shown in Fig. 2. Our system design partially follows previously described wide field FLIM endoscope instrumentation [43, 44], but here free space illumination using a diverging lens was used for conveniently exciting the leaf samples. The excitation source was a gainswitched diode laser (PicoQuant, LDH-P-C-440B with driver PDL-800-B), which provided pulses of $<500$ ps at $40 \mathrm{MHz}$ with average powers of $\sim 4 \mathrm{~mW}$. The emission from the diode laser was passed through a spectral clean-up filter (F1, Semrock, FF02-438/24) to suppress out of band radiation from the diode laser and the elliptical beam was expanded by a diverging lens to illuminate a FOV of $\sim 30 \times 6 \mathrm{~mm}$ on a leaf held in place by a clamp while attached to a live plant. A black anodized piece of metal was fixed to the clamp behind the leaf to block any unwanted signals or reflections. Neutral density filters were inserted inbetween the clean-up filter and diverging

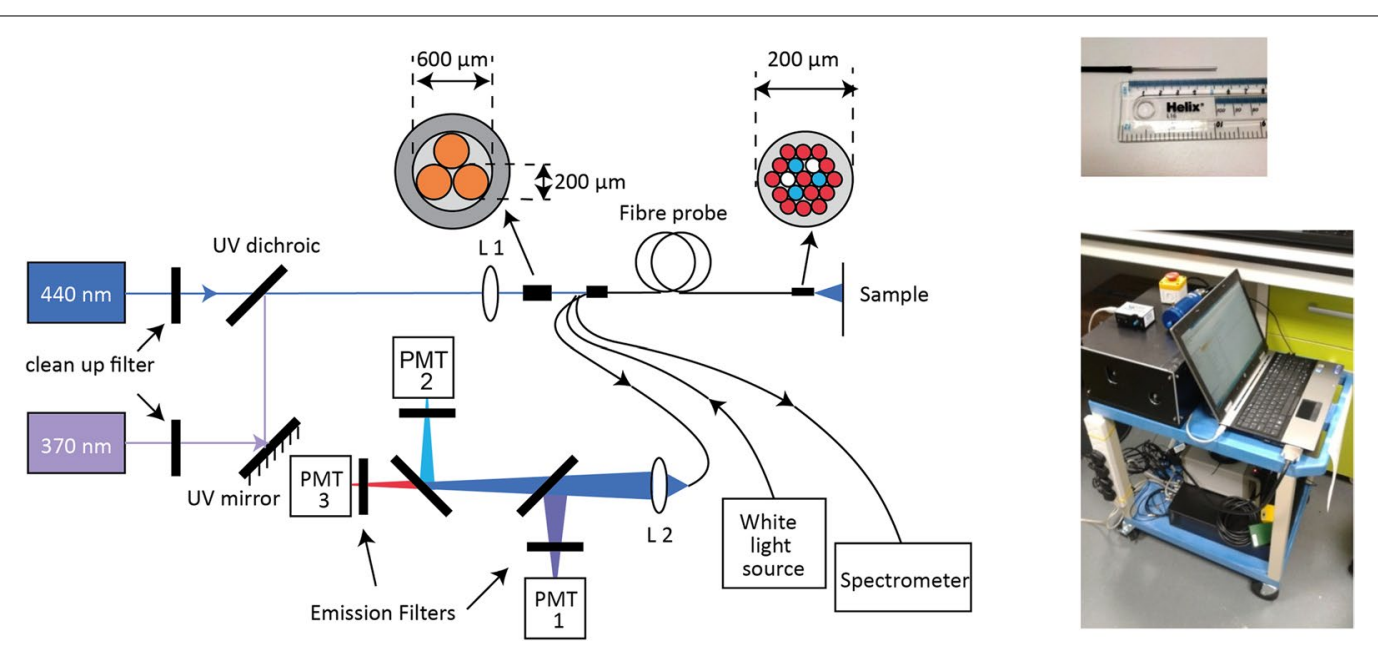

Fig. 1 Schematic diagram of portable multispectral time-resolved spectrofluorometer with fibre-optic probe with inset of probe tip and photograph of instrument 


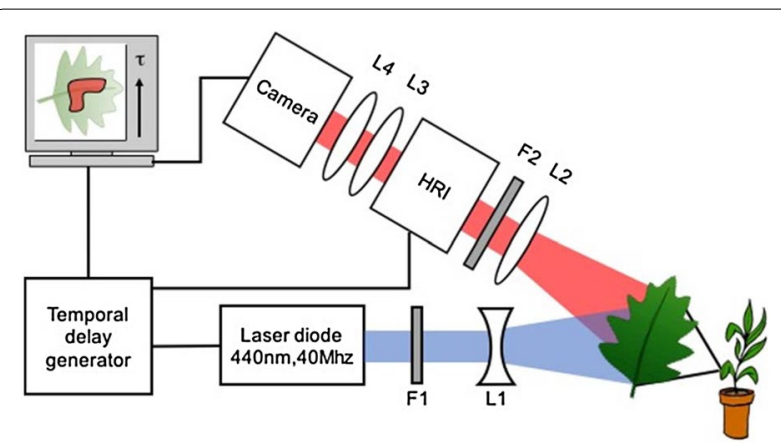

Fig. 2 Schematic diagram of the wide field imaging macroscope. $440 \mathrm{~nm}$ pulsed laser beam is passed through a cleanup filter $\mathrm{Fl}$ and expanded using a diverging lens, L1 to illuminate a leaf. Fluorescence from the leaf is collected by a camera lens, L2, and passed through an emission filter, F2, before it reaches a high rate imager (HRI). The collected signal is imaged on to a sCMOS camera by a pair of relay lenses $L 3, L 4$. The HRI is synchronized with the excitation laser via a temporal delay generator lens to reduce the excitation beam power to $0.3 \mathrm{~mW}$. The fluorescence from the leaf was collected by a camera lens (Pentax 12.5-75 $\mathrm{mm}$ ) and passed through an emission filter (F2, Semrock, 641/75 nm bandpass filter) chosen to overlap the chlorophyll emission peak at $690 \mathrm{~nm}$. The filtered fluorescence signal was focused onto a gated optical intensifier (Kentech Instruments, model HRI) read out by a sCMOS camera (Andor, Zyla 5.5) via two relay lenses (L3, L4). Wide-field time-gated FLIM, entails synchronizing the pulsed laser source with the HRI and adjusting the delay between excitation and time-gated detection to sample the fluorescence intensity decay profiles in each pixel. For this study we sampled the fluorescence signal with 9 time gates of $1 \mathrm{~ns}$ duration at increasing time delays after the excitation pulse. The read out camera was operated with an exposure time of $200 \mathrm{~ms}$ per frame. Overall, each FLIM acquisition required $\sim 3 \mathrm{~s}$.

The leaf sample was first imaged once without any treatment and then a time-series of FLIM images was acquired at $3 \mathrm{~min}$ intervals after application of two $2 \mu \mathrm{l}$ drops of Flagon EC 400. Image acquisition was controlled by the openHCA-FLIM $\mu$ Manager plug-in developed in the Photonics group at Imperial College London [45]. An IRF based on the excitation pulses was measured under the same experimental conditions using a scattering sample.

\section{Results}

\section{Plant autofluorescence characterization}

The fluorescence excitation-emission matrix for Triticum aestivum leaves measured using the Shimadzu spectrofluorometer is shown in Fig. 3. There are two major spectral emission bands: a blue-green fluorescence (ex $\sim 320 \mathrm{~nm}$, em $\sim 450 \mathrm{~nm}$ ) that could be due to the presence of cinnamic acids [46] and a fluorescence band attributed to chlorophyll with two prominent peaks at $690 \mathrm{~nm}$ and $720 \mathrm{~nm}$ [47]. For the subsequent fibre optic probe and wide-field imaging fluorescence lifetime measurements discussed below, we chose to concentrate on the $690 \mathrm{~nm}$ emission peak attributed to fluorescence from chlorophyll in leaves at $440 \mathrm{~nm}$ excitation as the spectral region of interest. This matched the capabilities of our instrumentation and we detected no significant fluorescence from the Flagon EC 400 in this spectral detection band, see below.

\section{Characterisation of Flagon EC $\mathbf{4 0 0}$ fluorescence}

The emission spectrum of Flagon EC 400 in the range 400-800 $\mathrm{nm}$ was measured (49 ppm solution) using the Shimadzu spectrofluorometer with excitation at $440 \mathrm{~nm}$, see Fig. 4a. The fluorescent decay profiles of Flagon EC 400 were measured ( $49 \mathrm{ppm}$ solution) using the custom-built multidimensional spectrofluorometer as described in Manning et al. [41] with excitation at $405 \mathrm{~nm}(20 \mathrm{MHz}$ repetition rate) and detection at $482 \mathrm{~nm}$. The data were fitted to a double exponential decay model using FLIMfit which returned lifetime components of 6214 ps (18\%) and 2239 ps (82\%) with an average $\chi^{2}$ value of 1.17 . An exemplar decay profile is shown in Fig. 4b. We investigated how the fluorescence decay profile varied across the emission spectrum

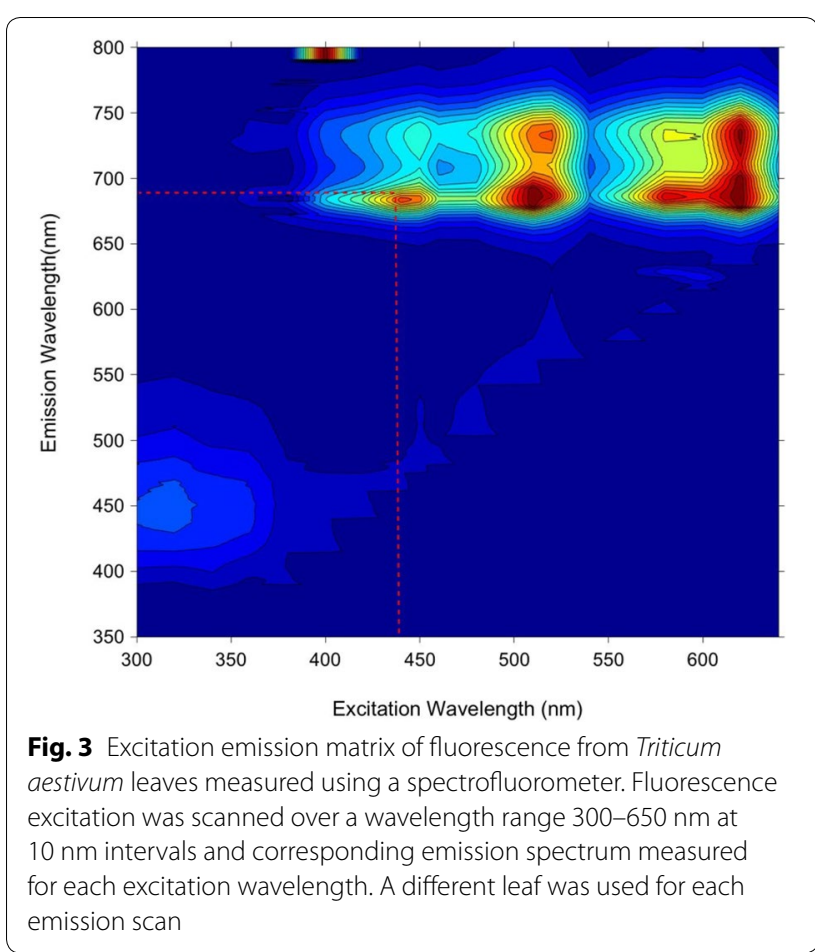



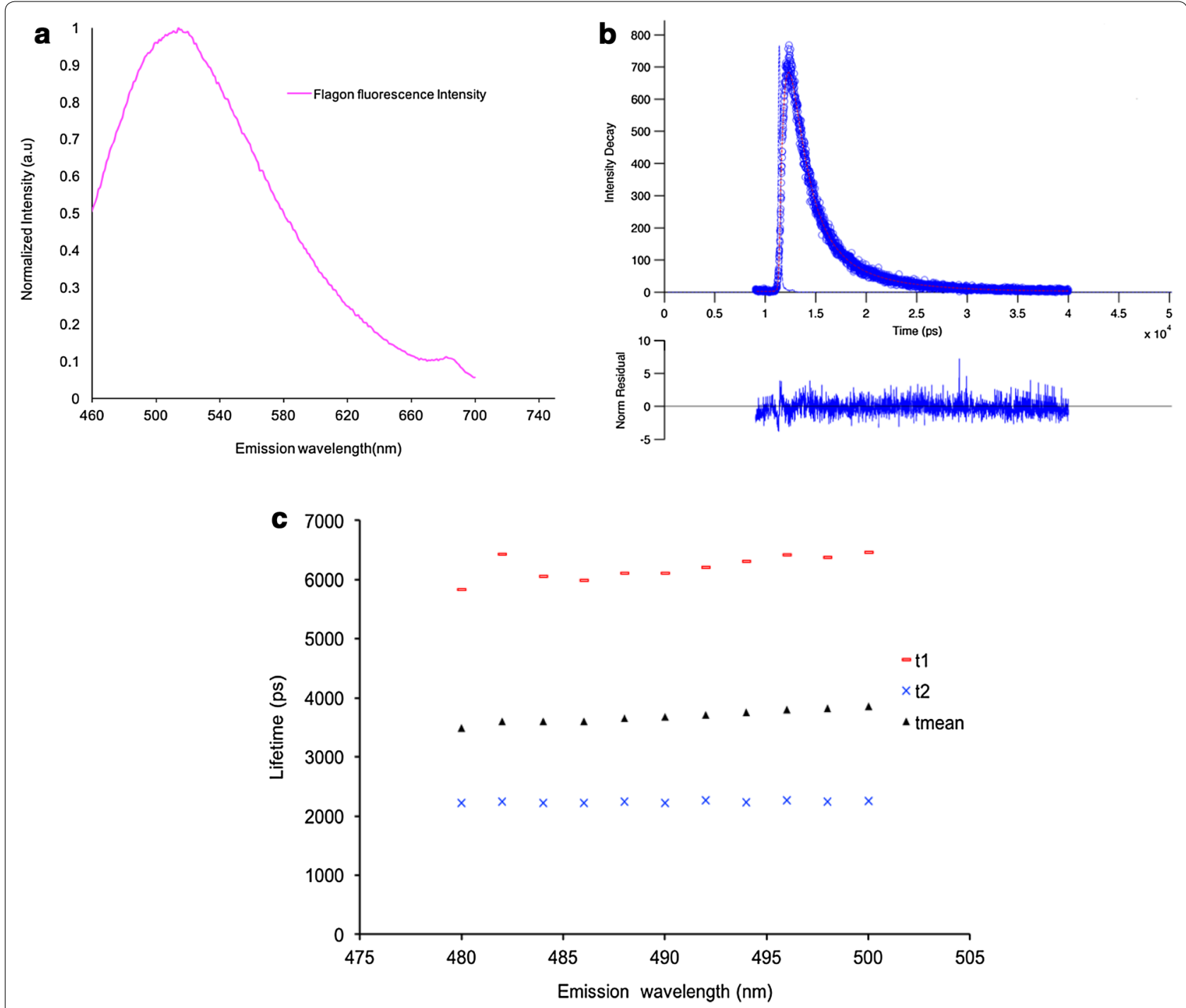

Fig. 4 a Fluorescence emission spectrum of Flagon EC 400 when excited at $440 \mathrm{~nm}$. b Fluorescence decay curve fitted with double exponential model using the FLIMfit software; $\mathbf{c}$ dependence of fluorescence lifetime on detection wavelength. Data shown in $\mathbf{b}$ and $\mathbf{c}$ was acquired with an excitation wavelength of $405 \mathrm{~nm}$

and the results are presented in Fig. 4c. Both lifetimes remain approximately constant over the detection range $480-500 \mathrm{~nm}$.

In order to show that fluorescence from Flagon EC400 is small compared to that from the Triticum aestivum leaf when using fluorescence excitation at $440 \mathrm{~nm}$, the two emission spectra were scaled according to the measurements of their relative emission intensities using the multispectral point-probe spectrofluorometer, see Fig. 5 . Here the emission intensity of Flagon EC 400 was measured when it was dried on to a black anodised aluminium surface. From this data the relative fluorescence signal from Flagon EC 400 is only $0.58 \%$ of chlorophyll fluorescence from a leaf obtained in the same spectral channel.
In vivo multiphoton FLIM showing effect of herbicide at a cellular level

To explore the indirect read out of the presence of the Flagon EC 400 herbicide, we used two photon excitation at $900 \mathrm{~nm}$ to acquire high resolution FLIM images of chlorophyll fluorescence in Triticum aestivum leaves. Imaging of treated (Flagon EC 400) and untreated plant leaves was performed in situ using TCSPC on the Leica SP5 multiphoton system with a detection spectral band from 600 to $730 \mathrm{~nm}$ and implemented with leaf samples from the same plant fixed to a microscope slide. We imaged 4 fields of view (FOVs) in the sample of untreated leaf and 4 FOVs in the sample of treated leaf at $2.5 \mathrm{~h}$ post treatment. Figure $6 \mathrm{a}, \mathrm{c}$ show typical autofluorescence 


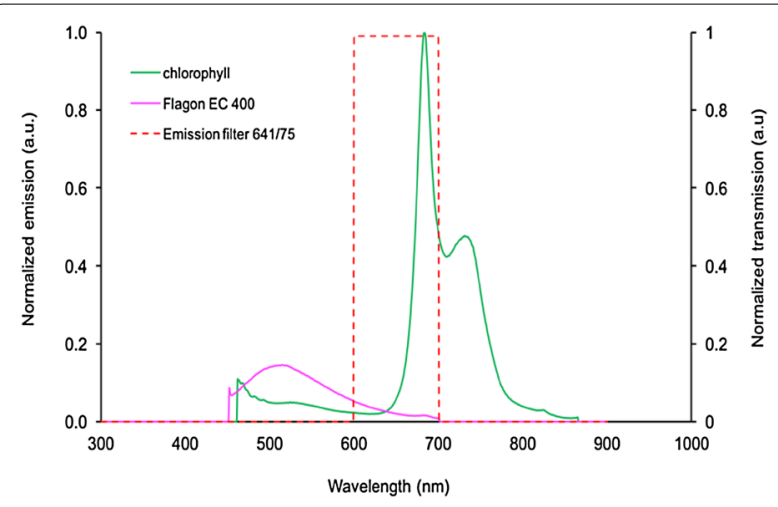

Fig. 5 Fluorescence emission spectra of chlorophyll and Flagon EC 400, when excited at $440 \mathrm{~nm}$ (curve normalized to maximum Flagon fluorescence). The transmission band of the emission filter used for wide-field FLIM is also plotted to show the spectral range of detection. The emission curves are scaled according to the data from control experiments carried out using the multispectral point-probe spectrofluorometer

intensity images and Fig. 6b, d show the corresponding intensity-merged fluorescence lifetime images of the untreated and treated leaves respectively. These images show mesophyll cells (larger ovoid shapes) and chloroplasts (smaller circular disc like structures) within them. It can be seen that the presence of the herbicide within the cells results in an increase in autofluorescence lifetime and an increase in the heterogeneity of the fluorescence lifetime. In the images shown in Fig. 6d, this heterogeneity is clearly visible across the different FOVs. These images were all acquired at a depth of $100 \mu \mathrm{m}$ from the leaf surface. Fluorescence decay profiles were fitted to a double exponential decay model, as in [29], using the FLIMfit software and the intensity-weighted mean lifetimes $\left(\tau_{m}\right)$ averaged over the FOV were calculated. This analysis indicated that the untreated FOVs exhibits an average $\tau_{m}$ of $560 \pm 30$ ps while the treated leaf sample exhibited increased $\tau_{m}$ of $2000 \pm 440$ ps across different FOVs as shown in Fig. 7, which is consistent with the chlorophyll fluorescence lifetime increasing in the presence of a PS II inhibiting herbicide.

Following these in situ fixed endpoint measurements, we undertook a time course of two photon excited FLIM (TPE-FLIM) measurements of treated and untreated plants in vivo. Figure 8a shows intensity merged fluorescence lifetime images of (a) an untreated leaf and (b) a treated leaf at different time points. As expected, the treated leaf exhibits an increase in the mean chlorophyll fluorescence lifetime in contrast to that of an untreated leaf.

A plot of the weighted mean lifetimes obtained from the analysis is presented in Fig. 9. The untreated leaf control time-course experiment yielded images with a $\tau_{m}$ of 351 ( $\pm 1 \mathrm{SE})$ ps averaged over the FOV and the time course of a treated leaf yielded images with an increased mean lifetime of 1021 ps at $5 \mathrm{~min}$ to 1110 ps at $45 \mathrm{~min}$ post treatment.

\section{In vivo multispectral point-probe autofluorescence lifetime measurements}

The multispectral fibre-optic point-probe was applied to in vivo studies of untreated and treated (with Flagon EC 400) leaves of Triticum aestivum, for which the results are shown in Fig. 10. The measured autofluorescence decay profiles were fitted to a double exponential model with the untreated leaves presenting a mean $\tau_{m}$ of 480 ( $\pm 16 \mathrm{SE})$ ps in the $440 \mathrm{~nm}$ excited chlorophyll channel, CH4 $(620-710 \mathrm{~nm})$. For leaves treated with Flagon EC 400, an increase in $\tau_{m}$ up to 1438 ps was observed in this channel.

In $\mathrm{CH} 3$, which detects fluorescence $(620-710 \mathrm{~nm})$ excited at $372 \mathrm{~nm}$ corresponding to the chlorophyll emission wavelengths, an average $\tau_{m}$ of $490( \pm 10$ S.E.) ps was obtained for untreated leaves and $1170( \pm 140$ S.E.) ps for treated leaves.

The autofluorescence decay profiles measured in $\mathrm{CH} 2$ (430-480 nm, excited at $372 \mathrm{~nm}$ ) presented a $\tau_{m}$ of $\sim 750 \mathrm{ps}$ for both treated and untreated leaves. This autofluorescence could be attributed to cinnamic acids or lignin [48].

Fluorescence collected from channel CH1 (400$420 \mathrm{~nm}$, excited at $372 \mathrm{~nm}$ ) is most strongly affected by background fluorescence from the optical fibres and does not follow a definite trend. The variation between measurements is attributed to the low signal level in this channel.

A time course experiment to study the changes in fluorescence lifetime after treating a leaf with Flagon EC 400 was undertaken and also analyzed using a biexponential fit model. The results are shown in Fig. 11. Treatment of the leaf with Flagon was carried out at time $=0$ and the time of the "untreated" leaf measurement was $5 \mathrm{~min}$ before treatment. In the first 5 min after treatment, $\tau_{m}$ is observed to have increased to $1 \mathrm{~ns}$ and then to $1.3 \mathrm{~ns}$ after $60 \mathrm{~min}$; thereafter it remained approximately constant up to $180 \mathrm{~min}$. Control experiments where the treatment, i.e. 2 drops of Flagon EC 400, was applied to a black anodized metal sheet resulted in no significant signal detected in the chlorophyll channel, $\mathrm{CH} 4$. The resultant fluorescence collected in $\mathrm{CH} 4$ from Flagon was found to be insignificant compared to the chlorophyll fluorescence as shown in Fig. 5. We studied the variations in fluorescence lifetimes in untreated lifetime in plants of different age groups and the weighted mean fluorescence lifetimes calculated from $\mathrm{CH} 4$ are shown in Additional 


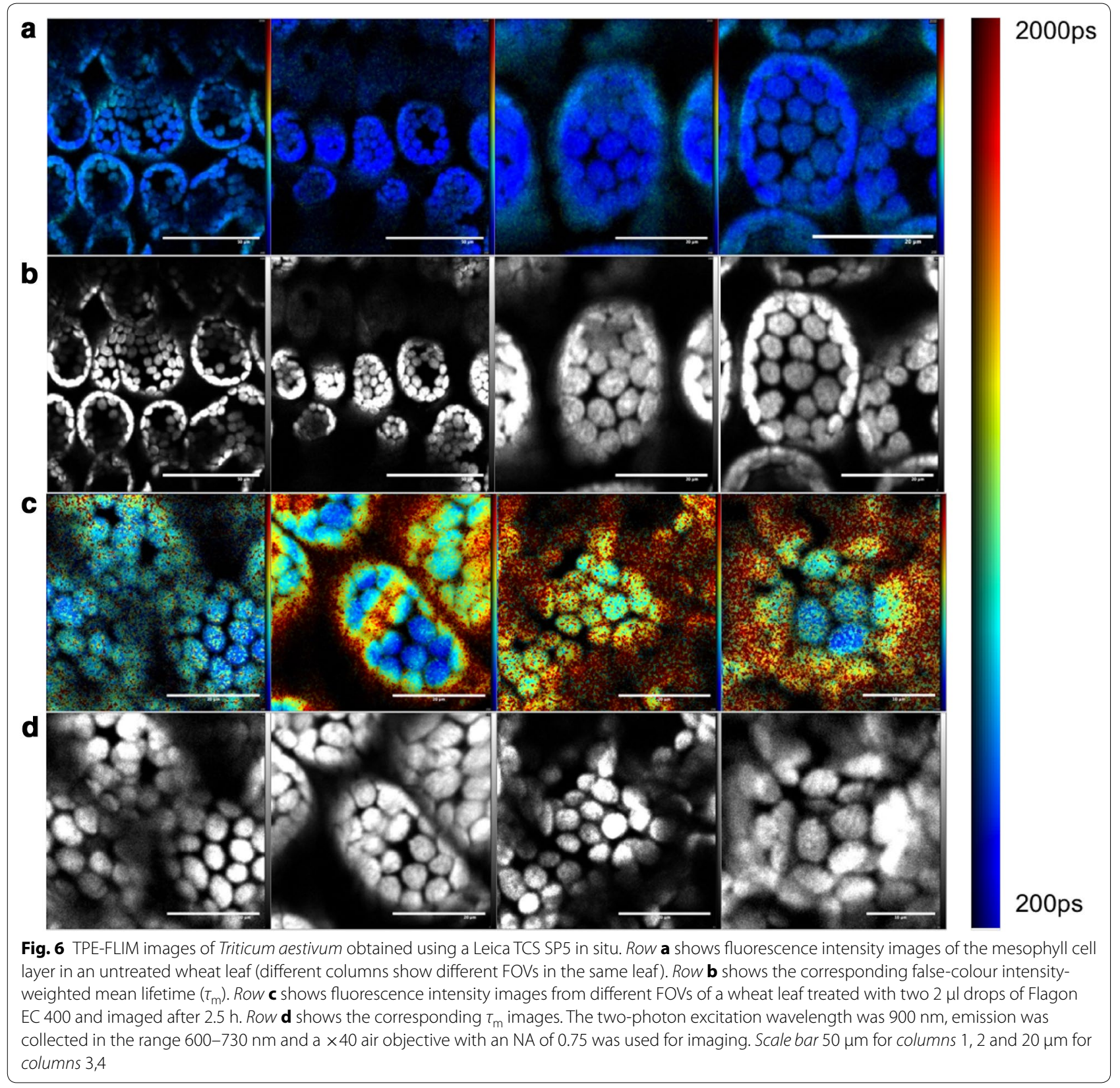

file 2. It can be seen that there are minor variations from plant to plant and in different age groups, but the general distribution could be seen to be lying in the $350-600$ ps range.

\section{Wide-field FLIM to map herbicide effect in Triticum aestivum}

In vivo wide-field time-gated FLIM of Triticum aestivum plant leaves before and following treatment with Flagon EC 400 was undertaken. To orientate this study, Fig. 12a shows a color photograph of the leaf with the treatment area (enclosed by the red-dotted circle) marked with four dots of a black felt pen. Figure 12b shows a fluorescence intensity image of the leaf before it is treated with Flagon EC 400. To confirm that there is no contribution to the fluorescent signal due to fluorescence of the herbicide, we performed a control experiment using the same treatment of Flagon EC 400 applied to a black anodized metal surface and did not detect any significant fluorescence. 


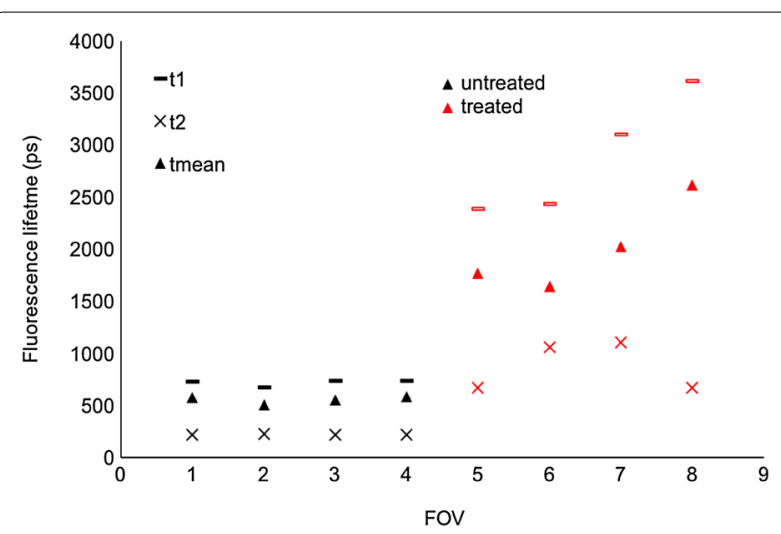

Fig. 7 Fluorescence lifetimes $\left(\tau_{\mathrm{m}}\right)$ calculated from different FOVs in treated and untreated leaves. $(1-4)$ Represent FOVs in the untreated leaf and (5-9) represents FOVs in a treated leaf. The data is obtained from the TPE-FLIM measurements shown in Fig. 6

Wide-field autofluorescence intensity and lifetime images were acquired of a leaf 3 min before treatment and then at $3 \mathrm{~min}$ intervals after the treatment up to $27 \mathrm{~min}$. The autofluorescence decay profiles were fitted pixel wise to a double exponential model using the FLIMfit software. Time-integrated autofluorescence intensity images and the corresponding intensitymerged autofluorescence lifetime images and lifetime histograms obtained from the experiment at each time point are presented in Fig. 13. The correlation plot shown in Fig. 14 shows 2D histograms of the autofluorescence intensity versus mean lifetime for the treated and untreated regions using the same data set as for Fig. 13. For the pixels with a short mean lifetime (associated with the untreated leaf) the plots show a vertical distribution of points indicating that there is no strong correlation between fluorescence intensity and fluorescence lifetime for this measurement.

Figure 15 presents the evolution of $\tau_{m}$ averaged over either the red-dashed region of interest (treated region) indicated in Fig. 12a or the segmented area of the leaf outside the red-dashed region of interest. The leaf presents an initial $\tau_{m}$ of 521 ps before treatment in the red-dashed region of interest. After the treatment with herbicide, the mean autofluorescence lifetime is observed to increase over time, as shown in Figs. 13 and 15. At 3 min after treatment, the mean lifetime was 1000 ps. At $27 \mathrm{~min}$ after treatment, $\tau_{m}$ averaged over the red-dashed region of interest was 2150 ps, whereas in the region outside the red dotted circle (untreated region), the fluorescence lifetime remained approximately constant with an average $\tau_{m}$ value of 530 ( \pm 10 S.E.) ps.

A separate time course experiment was conducted on a different untreated plant and a leaf was imaged under the same conditions and at intervals of $3 \mathrm{~min}$ and the

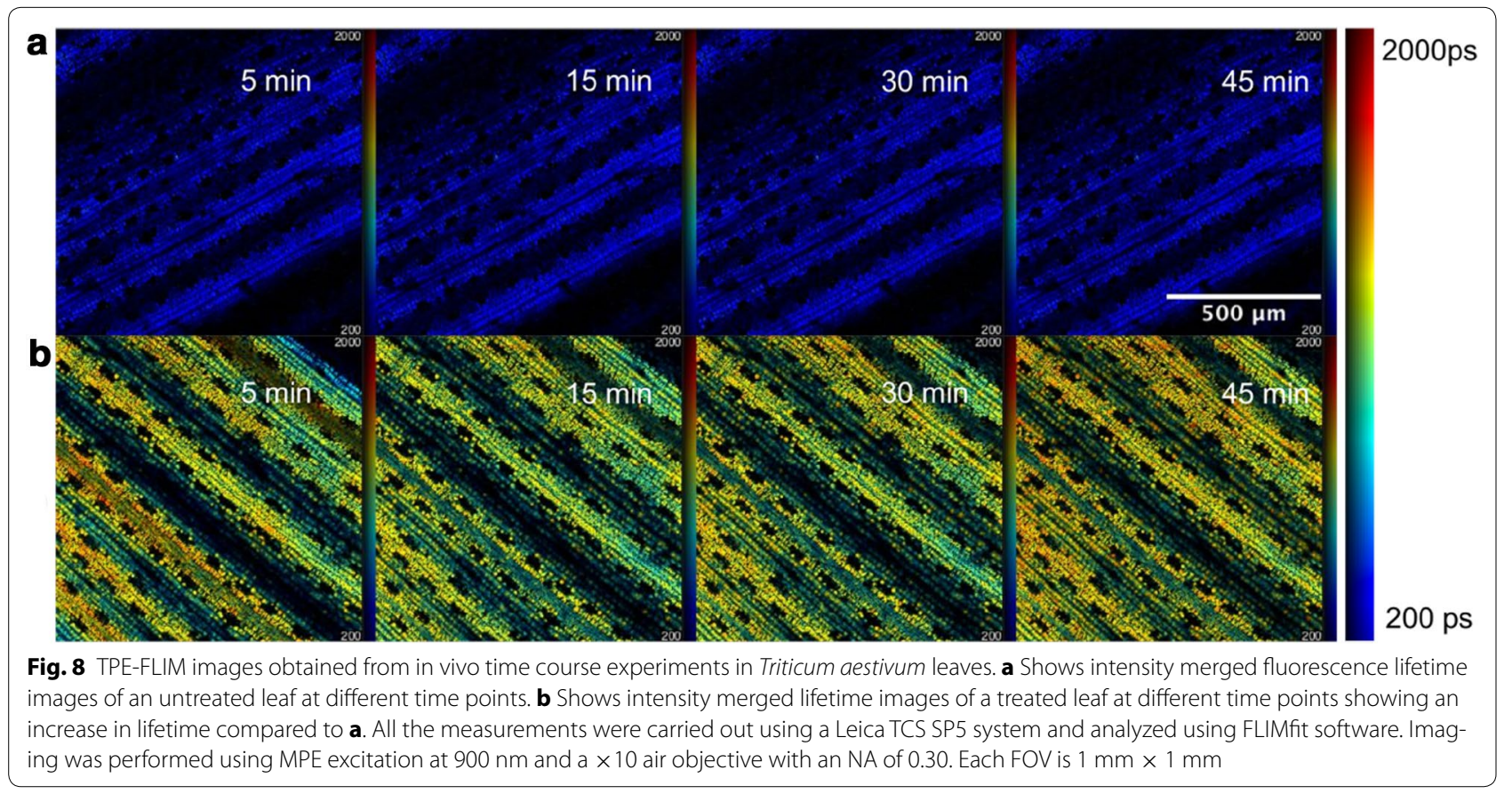




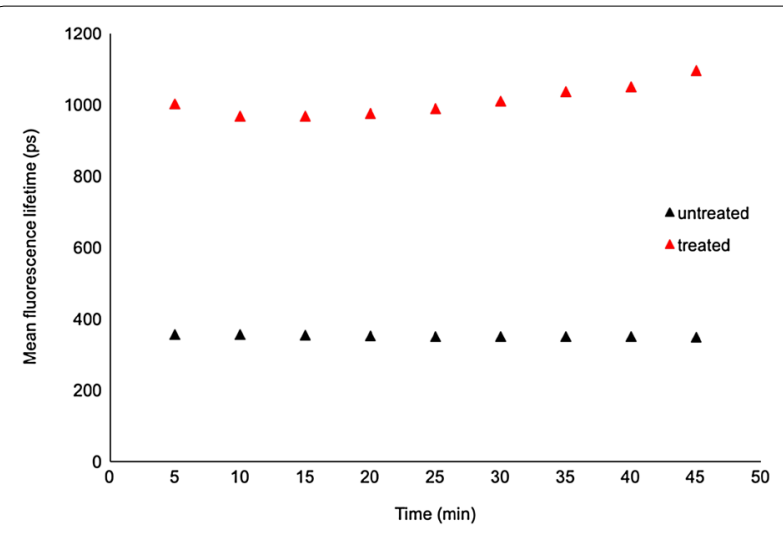

Fig. 9 Mean fluorescence lifetime $\left(\tau_{m}\right)$ plotted as a function of time for treated and untreated Triticum aestivum leaves imaged using multiphoton excitation at $900 \mathrm{~nm}$, see Fig. 8 for corresponding FLIM images. Standard errors are calculated from the pixelwise fit over the field of view shown in Fig. 8

results are shown in Fig. 16. The fluorescence lifetimes of the untreated leaf remained fairly constant over a time period of $27 \mathrm{~min}$ and yielded an average $\tau_{m}$ of $580( \pm 10$ S.E.) ps, in reasonable agreement with the $\tau_{m}$ values of the untreated regions of the treated leaf.
These lifetime values obtained are in agreement to the multiphoton imaging data. It can be seen from the lifetime frequency histograms that there is a significant decrease in the population fraction of the short lifetime ( 580 ps) component and the lifetime distribution shifts towards longer lifetimes (1000-2500 ps) over time after treatment.

Figure 16a shows the evolution over time of maps of the autofluorescence intensity and fluorescence lifetime of an untreated leaf imaged using wide field FLIM. Figure 16b shows the evolution over time of the mean fluorescence lifetime averaged over the whole of the leaf for the treated leaf presented in Fig. 13 and for the untreated leaf presented in Fig. 16a, again demonstrating the observation of a longer autofluorescence lifetime of chlorophyll in herbicide treated leaves.

\section{Discussion}

Although plant autofluorescence is highly complex and is not yet fully understood, this study indicates that it is possible to utilise lifetime measurements of autofluorescence attributed to chlorophyll to read out and map the action of the PS II inhibiting herbicide, Flagon EC 400. Our empirical in vivo measurements across multiphoton microscopy, point-probe lifetime measurements

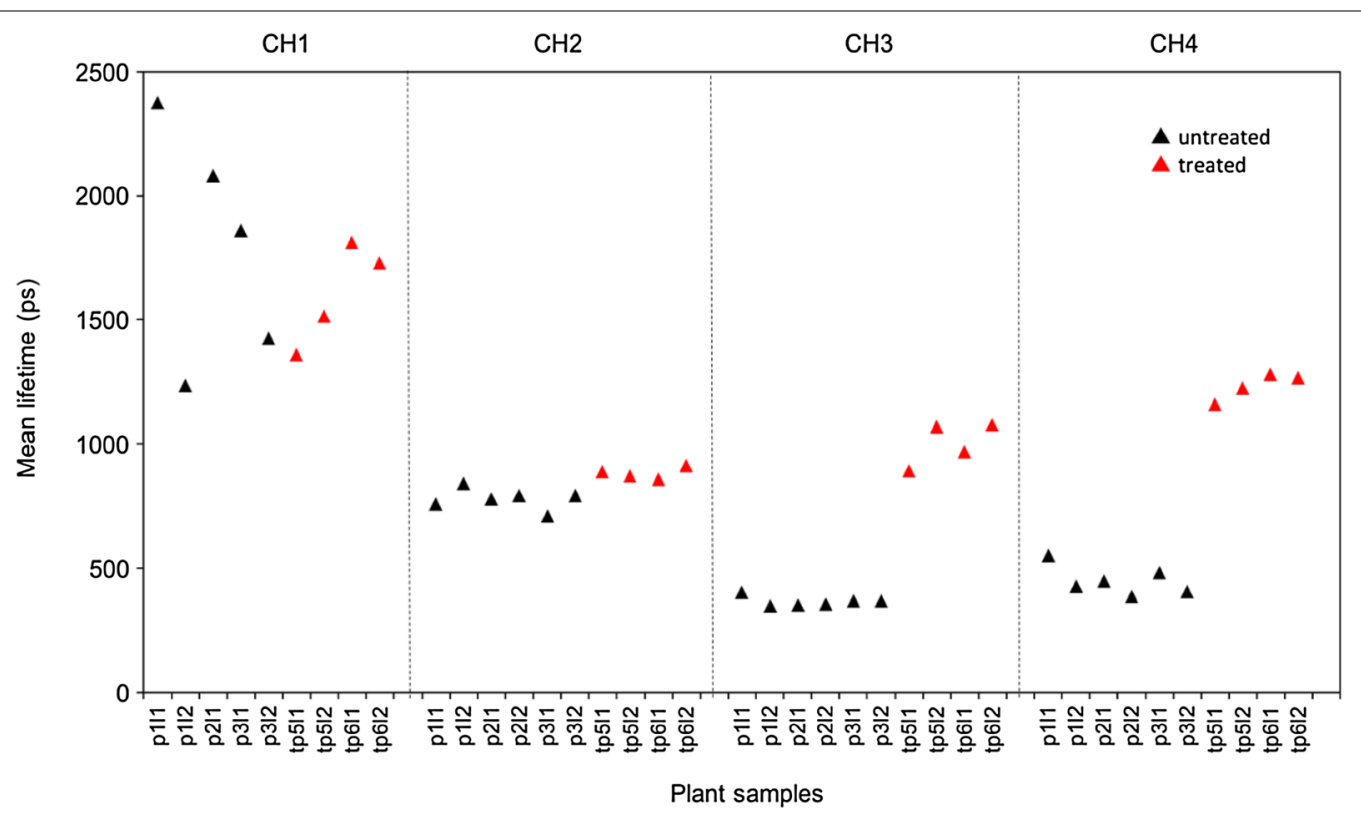

Fig. 10 Summary of multispectral lifetime point-probe measurements in Triticum aestivum. The different spectral detection channels are represented by $\mathrm{CH} 1, \mathrm{CH} 2, \mathrm{CH} 3$ and $\mathrm{CH} 4$ and are separated by black dotted vertical lines. Untreated plant samples are named in the format $\mathrm{p}(\mathrm{n})(\mathrm{m})$, where $\mathrm{n}$ is the plant number and $\mathrm{m}$ is the leaf number. Treated plants are named $\mathrm{tp}(\mathrm{n})(\mathrm{m})$. The weighted mean fluorescence lifetime $\left(\tau_{\mathrm{m}}\right)$ calculated for each sample is plotted in the respective spectral channels 


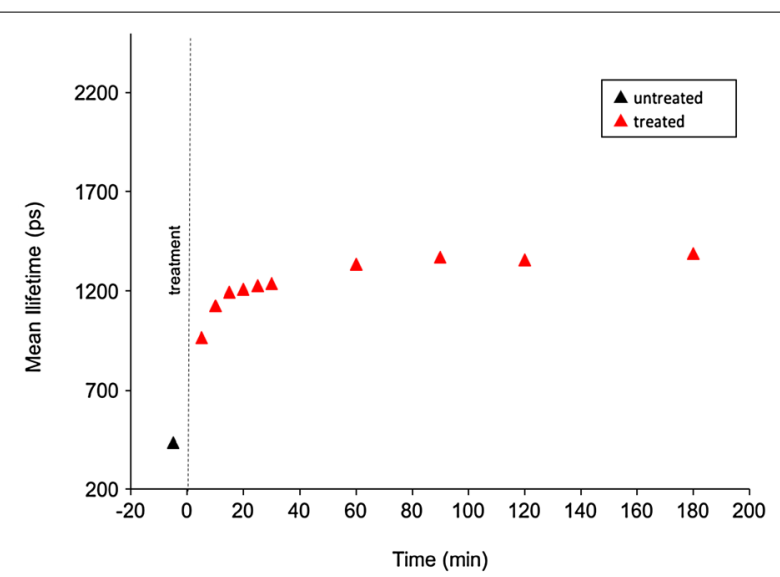

Fig. 11 Graph showing the changes in mean lifetime $\left(\tau_{m}\right)$ as a function of the time elapsed after treating the plant with Flagon EC 400. Black dotted line represents the time of treatment. Error bars show standard deviation calculated from 95\% confidence interval limits returned by FLIMfit software after fitting the single pixel data

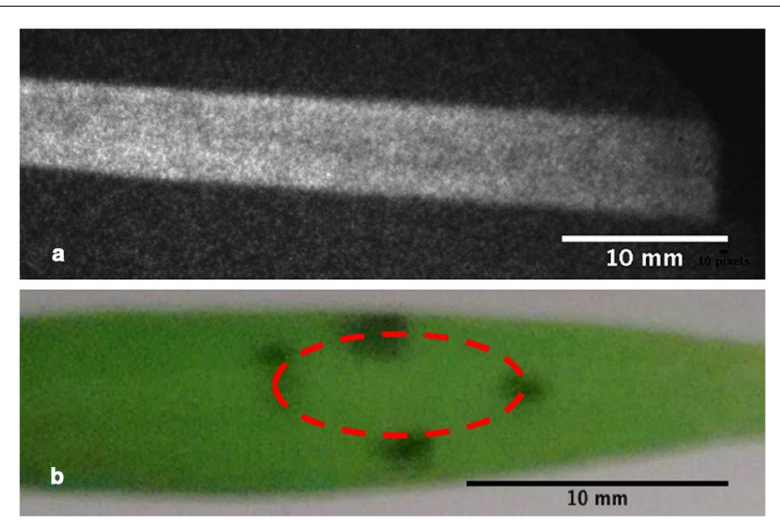

Fig. 12 a Color photograph of the portion of the leaf sample marked for treatment, the red dashed ellipse is a representation of the area to which the treatment spreads once it is applied on to the leaf. b Fluorescence intensity image of a leaf recorded by prior to treatment

and wide-field FLIM, all support the use of the change in lifetime of the autofluorescence in the chlorophyll emission spectral band to report the presence of the PS II herbicide through its localised impact on chlorophyll fluorescence. In the multiphoton microscopy images shown in Fig. 6d, a heterogeneity of lifetimes is clearly visible across the different FOVs. The $\tau_{m}$ values along the edges of the mesophyll cells are higher (Fig. 6d, especially the second, third and fourth images) compared to the insides of the cells. This could be explained by the active ingredient penetrating the cell membranes and inhibiting the PS II centres, which leads to an increased fluorescence lifetime of chlorophyll molecules in the vicinity of the corresponding herbicide binding sites, i.e. PS II centres. The observed increase in chlorophyll fluorescence lifetime in the presence of a PSII inhibitor may be explained by the blocking of energy transfer to this photosynthetic pathway and the consequently decreased rate of loss of energy through only the radiative and non-radiative decay channels. In principle, it should be possible to relate these observations to previous studies undertaken to model PS II [30] and light harvesting antenna complexes-II (LHC-II) $[49,50]$ but this would require disentangling contributions from different compartments in the leaf that are integrated in the analysis of our in vivo measurements. Multispectral multiphoton FLIM microscopy may enable this challenge to be addressed by segmenting different compartments in the leaf and will be the subject of future studies.

Our fluorescence measurement spectral windows from $\sim 600$ to $710 \mathrm{~nm}$ overlap with emission peaks from chlorophyll $a$ and chlorophyll $b$. However, since previous studies of energy transfer kinetics of photosynthetic pigments have indicated that chlorophyll $b$ molecules transfer most of their excitation energy to chlorophyll $a$ in less than 2 ps $[51,52]$, we believe that our measurements are dominated by chlorophyll $a$ fluorescence.

We note that these chlorophyll fluorescence lifetime measurements were undertaken with the leaf in a lightadapted state, for which the different light doses applied using the various methodologies are provided in the Additional file 3: Table S1. This indicates the potential of fluorescence lifetime measurements to provide quantitative readouts of the herbicide presence without subjecting the plant to dark adaptation, which avoids practical difficulties associated with spectral ratiometric measurements that do require dark adaption. We therefore see chlorophyll fluorescence lifetime measurements as a promising methodology for in field measurements.

We note that the distribution of herbicides could, in principle, be mapped by directly imaging the fluorescence 

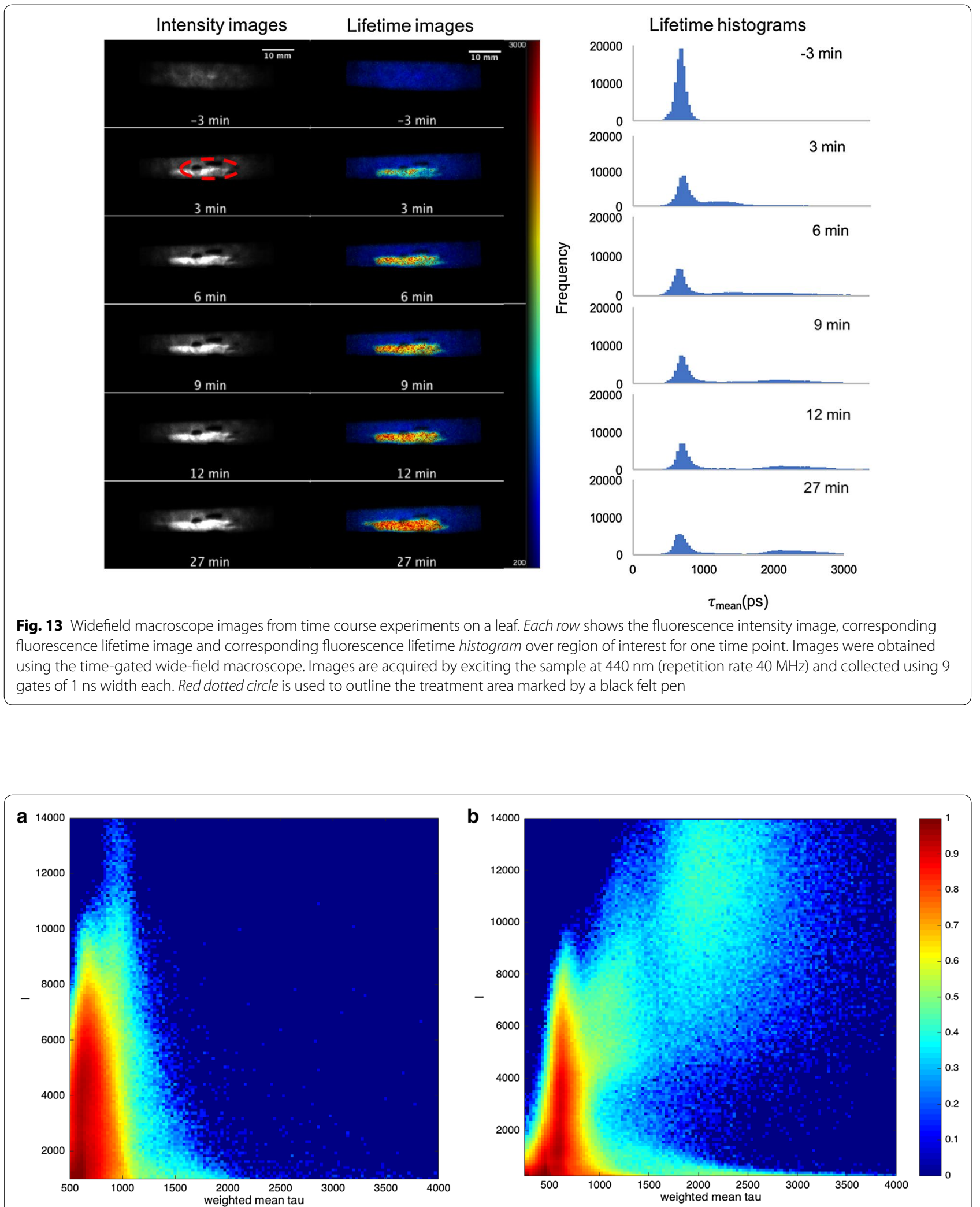

Fig. 14 Correlation plot of fluorescence intensity against weighted mean fluorescence lifetime for $\mathbf{a}$ the untreated region and $\mathbf{b}$ the treated region from the data acquired from all the time points after application of Flagon EC 400 using the wide field FLIM macroscope (same dataset as shown in Fig. 13) 


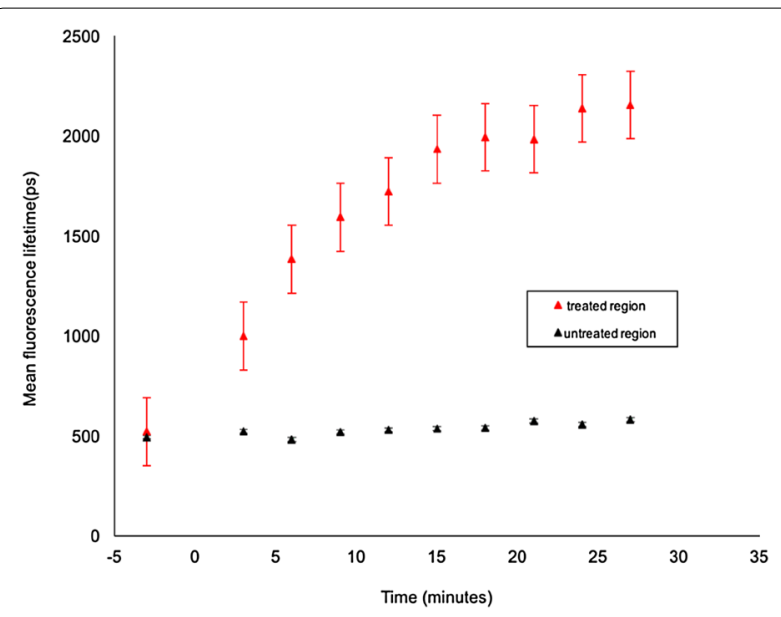

Fig. 15 Graph showing the changes in weighted mean lifetime, $\tau_{m}$ obtained from the wide field macroscope measurements as a function of the elapsed time after treating the plant with Flagon EC 400 for the region of interest marked with red dotted circles (treated region) and the region outside it (untreated region) separately. Black dotted line represents the time of treatment. Fluorescence lifetime calculations are made separately for the region of interest marked by the red dotted line and the region outside it. Standard errors are calculated from pixelwise fit over the region of interest of the herbicide but for most herbicides, including Flagon EC 400, this emission is very weak relative to the plant autofluorescence. As indicated in Fig. 3, the fluorescence of Flagon EC 400 is very weak in the chlorophyll detection band and we verified that no significant fluorescence signals were detected from the herbicide for the multispectral point probe measurements and the wide-field FLIM experiments.

\section{Conclusions}

We have demonstrated the in vivo readout of the local action of a PS-II inhibiting herbicide using multiphoton FLIM microscopy, a fibre-optically delivered timeresolved spectrofluorometer for single point lifetime measurements and single photon excited wide-field FLIM applied to leaves of Triticum aestivum. We consistently observed an increase in fluorescence lifetime in the chlorophyll autofluorescence detection band following treatment with Flagon EC 400 that can serve as an indirect marker of the herbicide action. The multispectral point probe system already has a form factor suitable to be used as a portable diagnostic tool in greenhouses and in the field. The wide-field FLIM instrument, which
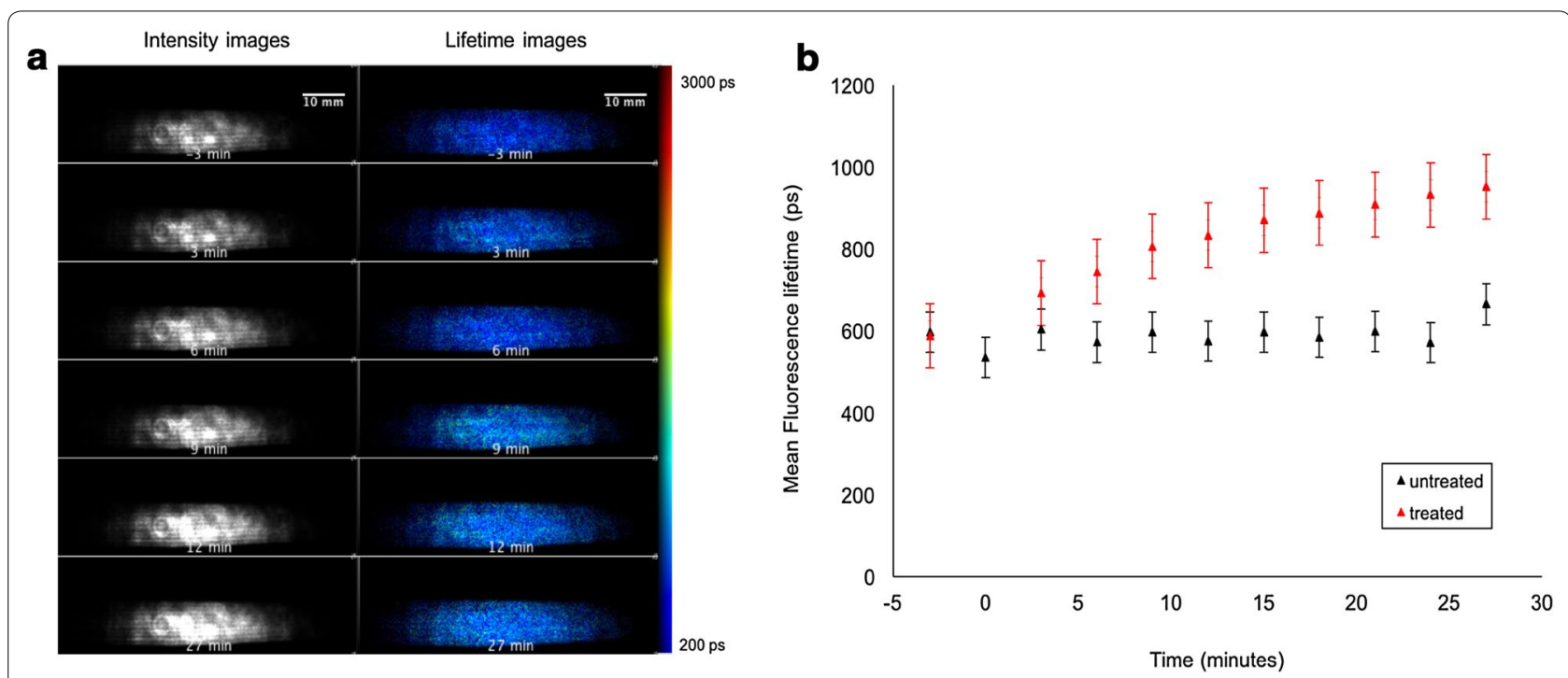

Fig. 16 a Widefield macroscope images from time course experiments on an untreated leaf. Each row shows the fluorescence intensity image and corresponding fluorescence lifetime images at a particular time point. b Changes in weighted mean lifetime, $\tau_{\mathrm{m}}$ averaged over the whole leaf obtained from the wide field macroscope measurements as a function of the elapsed time from an untreated life and a separate leaf treated with Flagon EC 400. Fluorescence lifetime values reported are the average over the entire image. Standard errors are calculated from pixelwise fit over the entire field of view 
enables spatiotemporal mapping of the distribution of this agrochemical over a large FOV comparable to an entire leaf, could also be engineered to be portable for in field studies.

\section{Additional files}

Additional file 1: Figure S1. Schematic representation of the optical set-up of multidimensional spectrofluorometer as described in Manning et al. [41].

Additional file 2: Figure S2. Distribution of fluorescence lifetimes in untreated Triticum aestivum plants of different age groups calculated from multispectral lifetime point-probe measurements in the spectral channel $\mathrm{CH} 4$ (excitation at $440 \mathrm{~nm}$, detection wavelengths $620-710 \mathrm{~nm}$ ). Data points from different age groups are represented by different colours. Plant samples are named in the format $p(n) \mid(m)$, where $n$ is the plant number and $m$ is the leaf number. The weighted mean fluorescence lifetime $\left(\tau_{m}\right)$ calculated for each sample is plotted here.

Additional file 3: Table S1. Table of light doses applied using the various methodologies.

\section{Abbreviations}

PS II: Photosystem II; FLIM: Fluorescence lifetime imaging; SEM: Scanning electron microscopy; CLSM: Confocal laser scanning microscopy; TPEM: Two-photon excitation microscopy; PS I: Photosystem I; DCMU: 3-(3,4-Dichlorophenyl)1,1-1-dimethylurea; Al: Active ingredient; TCSPC: Time-correlated single photon counting; IRF: Instrument response function; $\mathrm{CH}$ : Channel 1; $\mathrm{CH} 2$ : Channel 2; CH3: Channel 3; CH4: Channel 4; FOV: Field of view; TPE-FLIM: Two photon excited FLIM; LHC-II: Light harvesting antenna complexes-II.

\section{Authors' contributions}

EN, CS, CD and PF designed the experiments, which were performed by EN, SK and FG; EN analysed the data supervised by $C D$ and PF; EN, CD and PF wrote the manuscript. All authors read and approved the final manuscript.

\section{Author details}

1 Photonics Group, Department of Physics, Imperial College London, London SW7 2AZ, UK. ${ }^{2}$ Department of Chemistry, Imperial College London, London SW7 2AZ, UK. ${ }^{3}$ Institute of Chemical Biology, Imperial College London, London SW7 2AZ, UK. ${ }^{4}$ Syngenta, Jealott's Hill International Research Centre, Bracknell, Berkshire RG42 6EY, UK. ${ }^{5}$ Centre for Pathology, Imperial College London, London SW7 2AZ, UK

\section{Acknowledgements}

We acknowledge research funding from the European Union Seventh Framework Programme (FP7/2007-2013) under Grant Agreement Number 607466, the UK Biotechnology and Biological Sciences Research Council (BBSRC, BB/ M006786/1) and the Engineering and Physical Sciences Research Council (EPSRC) grant EP/I02770X/1. FG acknowledges a PhD studentship from the Engineering and Physical Sciences Research Council. Seeds of Triticum aestivum (Winter Wheat cv. Hereward) and Flagon EC 400 were obtained from Syngenta Jealott's Hill International Research Centre Bracknell, Berkshire, UK.

\section{Competing interests}

The authors declare that they have no competing interests.

\section{Availability of data and materials}

The datasets used and/or analysed during the current study available from the corresponding author on reasonable request.

\section{Funding}

The research leading to these results has received funding from the European Union Seventh Framework Programme (FP7/2007-2013) part of Marie (Skłodowska-)Curie actions research fellowships under grant agreement number 607466, the UK Biotechnology and Biological Sciences Research Council (BBSRC, BB/M006786/1) and the UK Engineering and Physical Sciences
Research Council (EPSRC, EP/I02770X/1). FG acknowleddges a Ph.D. studentship from the EPSRC.

\section{Publisher's Note}

Springer Nature remains neutral with regard to jurisdictional claims in published maps and institutional affiliations.

Received: 21 December 2016 Accepted: 8 June 2017

Published online: 15 June 2017

\section{References}

1. Wild E, Dent J, Thomas GO, Jones KC. Real-time visualization and quantification of PAH photodegradation on and within plant leaves. Environ Sci Technol. 2005:39:268-73.

2. Mullen AK, Clench MR, Crosland S, Sharples KR. Determination of agrochemical compounds in soya plants by imaging matrix-assisted laser desorption/ionisation mass spectrometry. Rapid Commun Mass Spectrom. 2005:19:2507-16.

3. Sarsby J, Towers MW, Stain C, Cramer R, Koroleva OA. Mass spectrometry imaging of glucosinolates in arabidopsis flowers and siliques. Phytochemistry. 2012;77:110-8

4. Kim J, Jung J, Lee C. In vivo monitoring of the incorporation of chemicals into cucumber and rice leaves by chlorophyll fluorescence imaging. Plant Biotech. 2002:4:173-9.

5. Siminszky B, Corbin FT, Ward ER, Fleischmann TJ, Dewey RE. Expression of a soybean cytochrome P450 monooxygenase CDNA in yeast and tobacco enhances the metabolism of phenylurea herbicides. Proc Natl Acad Sci. 1999;96:1750-5.

6. Yilmaz G, Dane F. Phytotoxicity induced by herbicide and surfactant on stomata and epicuticular wax of wheat. Rom Biotechnol Lett. 2012:17:7757-65.

7. Lichtenthaler HK, Lang M, Sowinska M, Summ P, Heisel F, Miehe JA. Uptake of the herbicide diuron as visualised by the fluorescence imaging technique. Bot Acta. 1997;1 10:158-63.

8. Hepler PK, Gunning BES. Confocal fluorescence microscopy of plant cells, Protoplasma. 1998;201:121-57.

9. Hutzler P, Fischbach R, Heller W, Jungblut TP, Reuber S, Schmitz R, Veit M, Weissenbock G, Schnitzler J-P. Tissue localization of phenolic compounds in plants by confocal laser scanning microscopy. J Exp Bot. 1998:49:953-65.

10. Wild E, Dent J, Thomas GO, Jones KC. Use of two-photon excitation microscopy and autofluorescence for visualizing the fate and behavior of semivolatile organic chemicals within living vegetation. Environ Toxicol Chem. 2007:26:2486-93.

11. Feijó JA, Moreno N. Imaging plant cells by two-photon excitation. Protoplasma. 2004:223:1-32.

12. Maxwell K, Johnson GN. Chlorophyll fluorescence-a practical guide. J Exp Bot. 2000;51:659-68.

13. Holub O, Seufferheld MJ, Gohlke C, Govindjee, Heiss GJ, Clegg RM. Fluorescence lifetime imaging microscopy of Chlamydomonas reinhardtii: non-photochemical quenching mutants and the effect of photosynthetic inhibitors on the slow chlorophyll fluorescence transient. J Microsc 2007:226(Pt 2):90-120.

14. Fuerst EP, Norman MA. Interactions of herbicides with photosynthetic electron transport. Weed Sci. 1991:39:458-64.

15. Perkins RG, Oxborough K, Hanlon ARM, Underwood GJC, Baker NR. Can chlorophyll fluorescence be used to estimate the rate of photosynthetic electron transport within microphytobenthic biofilms? Mar Ecol Prog Ser. 2002:228:47-56.

16. Baker NR. Chlorophyll fluorescence: a probe of photosynthesis in vivo. Annu Rev Plant Biol. 2008;59:89-113.

17. Barbagallo RP, Oxborough K, Pallett KE, Baker NR. Rapid, noninvasive screening for perturbations of metabolism and plant growth using chlorophyll fluorescence imaging. Plant Physiol. 2003;132:485-93.

18. Agati G, Cerovic ZG, Moya I. The effect of decreasing temperature up to chilling values on the in vivo F685/F735 chlorophyll fluorescence ratio in Phaseolus vulgaris and Pisum sativum: the role of the photosystem i 
contribution to the $735 \mathrm{~nm}$ fluorescence band \&\#xB6. Photochem Photobiol. 2000;72:75-84.

19. Baker NR, Rosenqvist E. Applications of chlorophyll fluorescence can improve crop production strategies: an examination of future possibilities. J Exp Bot. 2004;55:1607-21.

20. Baker NR, Oxborough K. Chlorophyll fluorescence as a probe of photosynthetic productivity. In: Papageorgiou GC, Govindjee, editors. Chlorophyll a fluorescence: a signature of photosynthesis. Dordrecht: Springer 2004. p. 65-82.

21. Mishra KB, Mishra A, Novotná K, Rapantová B, Hodaňová P, Urban O, Klem K, Somerville C, Briscoe L, Lobell D, Schlenker W, Costa-Roberts J, AghaKouchak A, Feldman D, Hoerling M, Huxman T, Lund J, Thomson J, Valliyodan B, Nguyen H, Boyer J, Campbell B, Bray E, Gargallo-Garriga A, Sardans J, Pérez-Trujillo M, Oravec M, Urban O, Jentsch A, Kreyling J, et al. Chlorophyll a fluorescence, under half of the adaptive growth-irradiance, for high-throughput sensing of leaf-water deficit in Arabidopsis thaliana accessions. Plant Methods. 2016;12:46.

22. Mishra KB, Mishra A, Klem K. Govindjee: plant phenotyping: a perspective. Indian J Plant Physiol. 2016;21:514-27.

23. Lichtenthaler HK, Miehe JA. Fluorescence imaging as a diagnostic tool for plant stress. Elsevier Sci. 1997;2:6-10.

24. Lichtenthaler HK, Langsdorf G, Buschmann C. Uptake of diuron and concomitant loss of photosynthetic activity in leaves as visualized by imaging the red chlorophyll fluorescence. Photosynth Res. 2013;116:355-61.

25. Pawley JB, editor. Handbook of biological confocal microscopy. 3rd ed. Boston: Springer; 2006.

26. Broess K, Borst JW, van Amerongen H. Applying two-photon excitation fluorescence lifetime imaging microscopy to study photosynthesis in plant leaves. Photosynth Res. 2009;100:89-96.

27. Lakowicz JR, editor. Principles of fluorescence spectroscopy. $3 \mathrm{rd} \mathrm{ed}$. Boston: Springer; 2006.

28. Marcu L, French PMW, Elson DS, editors. Fluorescence lifetime spectroscopy and imaging: principles and applications in biomedical diagnostics. Boca Raton: CRC Press; 2014.

29. Holub O, Seufferheld MJ, Gohlke C, Govindjee, Clegg RM. Holub Clegg fluorescnce lifetime imaging in real time- a new technique for photosynthesis research 2000.pdf. Photosynthetica. 2000;38:581-99.

30. Iwai M, Yokono M, Inada N, Minagawa J. Live-cell imaging of photosystem II antenna dissociation during state transitions. Proc Natl Acad Sci. 2010:107:2337-42.

31. Babourina O, Rengel Z. Uptake of aluminium into Arabidopsis root cells measured by fluorescent lifetime imaging. Ann Bot. 2009;104:189-95.

32. Hotzer B, Ivanov R, Bauer P, Jung G. Investigation of copper homeostasis in plant cells by fluorescence lifetime imaging microscopy. Plant Signal Behav. 2012;7(April):521-3.

33. Petrásek Z, Schmitt F-J, Theiss C, Huyer J, Chen M, Larkum A, Eichler HJ, Kemnitz K, Eckert H-J. Excitation energy transfer from phycobiliprotein to chlorophyll $\mathrm{d}$ in intact cells of Acaryochloris marina studied by time- and wavelength-resolved fluorescence spectroscopy. Photochem Photobiol Sci. 2005:4:1016-22.

34. Hunsche M, Bürling K, Noga G. Spectral and time-resolved fluorescence signature of four weed species as affected by selected herbicides. Pestic Biochem Physiol. 2011;101:39-47.

35. Xu C, Zipfel W, Shear JB, Williams RM, Webb WW. Multiphoton fluorescence excitation: new spectral windows for biological nonlinear microscopy. Proc Natl Acad Sci. 1996;93:10763-8.

36. Littlejohn GR, Mansfield JC, Christmas JT, Witterick E, Fricker MD, Grant MR, Smirnoff N, Everson RM, Moger J, Love J. An update: improvements in imaging perfluorocarbon-mounted plant leaves with implications for studies of plant pathology, physiology, development and cell biology. Front Plant Sci. 2014;5(April):140.

37. Lagarto J, Dyer BT, Talbot C, Sikkel MB, Peters NS, French PMW, Lyon AR, Dunsby C. Application of time-resolved autofluorescence to label-free in vivo optical mapping of changes in tissue matrix and metabolism associated with myocardial infarction and heart failure. Biomed Opt Express. 2015;6:324-46.

38. McGinty J, Galletly NP, Dunsby C, Munro I, Elson DS, Requejo-Isidro J, Cohen P, Ahmad R, Forsyth A, Thillainayagam AV, Neil MAA, French PMW Stamp GW. Wide-field fluorescence lifetime imaging of cancer. Biomed Opt Express. 2010;1:627.
39. Cerovic ZG, Nicolas FM, Moya I. Ultraviolet-induced fluorescence for plant present state and prospects monitoring. Agronomie. 1999;19:543-78.

40. Meyer S. UV-induced blue-green and far-red fluorescence along wheat leaves: a potential signature of leaf ageing. J Exp Bot. 2003;54:757-69.

41. Manning HB, Kennedy GT, Owen DM, Grant DM, Magee Al, Neil MAA, Itoh Y, Dunsby C, French PMW. A compact, multidimensional spectrofluorometer exploiting supercontinuum generation. J Biophoton. 2008;1:494-505.

42. Warren SC, Margineanu A, Alibhai D, Kelly DJ, Talbot C, Alexandrov Y, Munro I, Katan M, Dunsby C, French PMW. Rapid global fitting of large fluorescence lifetime imaging microscopy datasets. PLoS One. 2013;8:e70687.

43. Sparks H, Warren S, Guedes J, Yoshida N, Charn TC, Guerra N, Tatla T, Dunsby C, French P. A flexible wide-field FLIM endoscope utilising blue excitation light for label-free contrast of tissue. J Biophoton. 2015:8:168-78.

44. Requejo-Isidro J, Mcginty J, Munro I, Elson DS, Galletly NP, Lever MJ, Neil MAA, Stamp GWH, French PMW, Kellett PA, Hares JD. High-speed widefield time-gated endoscopic fluorescence-lifetime imaging. Opt Lett. 2004;29:2249-51.

45. OpenFLIM-HCA Acquisition Software. http://www.imperial.ac.uk/ photonics/research/biophotonics/instruments-software/openflim-hca/.

46. Harris PJ, Hartley RD. Detection of bound ferulic acid in cell walls of the Gramineae by ultraviolet fluorescence microscopy. Nature. 1976;259:508-10.

47. Rabinowitch E, Govindjee. Photosynthesis. New York: Wiley; 1969.

48. Coble PG, Lead J, Baker A, Reynolds DM, Spencer RG. Aquatic organic matter fluorescence. Cambridge: Cambridge University Press; 2014.

49. Pascal AA, Liu Z, Broess $K$, van Oort B, van Amerongen $H$, Wang $C$, Horton P, Robert B, Chang W, Ruban A. Molecular basis of photoprotection and control of photosynthetic light-harvesting. Nat Lett. 2005:436:134-7.

50. Leupold D, Teuchner K, Ehlert J, Irrgang K-D, Renger G, Lokstein H. Twophoton excited fluorescence from higher electronic states of chlorophylls in photosynthetic antenna complexes: a new approach to detect strong excitonic chlorophyll a/b coupling. Biophys J. 2002;82:1580-5.

51. Croce R, Müller MG, Bassi R, Holzwarth AR. Chlorophyll b to chlorophyll a energy transfer kinetics in the CP29 antenna complex: a comparative femtosecond absorption study between native and reconstituted proteins. Biophys J. 2003;84:2508-16.

52. Gradinaru CC, van Stokkum IHM, Pascal AA, van Grondelle R, van Amerongen $\mathrm{H}$. Identifying the pathways of energy transfer between carotenoids and chlorophylls in LHCII and CP29. A multicolor, femtosecond pump-probe study. J Phys Chem B. 2000;104:9330-42.

\section{Submit your next manuscript to BioMed Central and we will help you at every step:}

- We accept pre-submission inquiries

- Our selector tool helps you to find the most relevant journal

- We provide round the clock customer support

- Convenient online submission

- Thorough peer review

- Inclusion in PubMed and all major indexing services

- Maximum visibility for your research

Submit your manuscript at www.biomedcentral.com/submit 